\title{
Tendências em Séries Temporais de Precipitação no Norte do Estado do Rio Grande do Sul, Brasil
}

\author{
Hugo Alexandre Soares Guedes ${ }^{1}$ (D), Priscila dos Santos Priebe ${ }^{1}$, Emanuele Baifus Manke $^{1}$ \\ ${ }^{I}$ Programa de Pós-Graduação em Recursos Hídricos, Centro de Desenvolvimento Tecnológico, \\ Universidade Federal de Pelotas, Pelotas, RS, Brasil.
}

Recebido em: 3 de Outubro de 2015 - Aceito em: 10 de Dezembro de 2018

\begin{abstract}
Resumo
O objetivo deste estudo foi relacionar as tendências de aumento e/ou decréscimo em séries temporais de precipitação na metade norte do estado do Rio Grande do Sul, Brasil, com as fases do ENOS. Foram utilizadas séries temporais de dados pluviométricos de oito estações meteorológicas. Os dados de precipitação total anual foram previamente analisados e suas falhas preenchidas. Em seguida, foram plotados em forma de gráfico, juntamente com a média e a regressão linear. As séries pluviométricas também foram analisadas no software TREND, aplicando os testes estatísticos não paramétricos Mann-Kendall e Spearman's rho. Os testes identificaram alterações na tendência de 50\% das estações analisadas, corroborando com os resultados encontrados por meio de gráficos. O aumento da precipitação total anual nas séries temporais pode estar relacionado com os períodos de El Niño e La Niña. Entretanto, estudos mais específicos devem ser realizados para confirmar essa hipótese, uma vez que a variabilidade de precipitação também pode estar relacionada com alterações hidrológicas, em função do uso e ocupação do solo, e com atividades antrópicas na região norte do Estado.
\end{abstract}

Palavras-chave: El Niño, La Niña, mudanças climáticas.

\section{Precipitation Trends on Time Series in North of Rio Grande do Sul State, Brazil}

\begin{abstract}
The aim of this paper is relating trends of increasing and/or decreasing on precipitation time series in the north part of Rio Grande do Sul state, Brazil, with ENOS phases. To do so, time series of rainfall data from eight meteorological stations were used. The annual total precipitation data were previously analyzed and their gaps were filled. Then, they were plotted in charts, as well as the average data and the linear regression. Pluviometrical series were also analyzed using the software TREND, applying the following non-parametrical statistical tests: Man-Kendall and Spearman's rho. Tests identified changes on trends in $50 \%$ of the analyzed stations, corroborating with results found in charts. Increase of the annual total precipitation in temporal series may be related with El Niño and La Niña periods. However, more specific studies should be performed in order to confirm this hypothesis, since rainfall variability may also be related with hydrological changes, as a result of use and occupation of the soil as well as anthropic activities in the north part of the state.
\end{abstract}

Keywords: El Niño, La Niña, climate changes.

\section{Introdução}

As alterações climáticas e suas implicações para a humanidade têm sido alvo de muitas pesquisas da comunidade científica nos últimos anos, principalmente em relação às tendências de aumento e decréscimo nas séries históricas de precipitação (Ahmad et al., 2015; Ribeiro et al., 2014; Duhan e Pandey, 2013; Cera e Ferraz, 2015) e à variabilidade climática (Ávila et al., 2014; Polzin e

Autor de correspondência: Hugo Alexandre Soares Guedes, hugo.hydro@gmail.com. 
Hastenrath, 2014; Cera e Ferraz, 2015; Oliveira e Silva, 2016). De acordo com Moreira e Naghettini (2016) identificar e quantificar estas alterações é um processo complexo, dada a incerteza presente na dinâmica dos processos hidrológicos.

Por ser determinante na escolha de culturas e por influenciar as alterações ecológicas dos tipos de grãos a serem utilizados, a precipitação é uma das mais importantes variáveis hidrológicas (Duhan e Pandey, 2013). Em vários países do mundo foram detectadas alterações nas tendências pluviométricas, podendo-se citar o Paquistão (Ahmad et al., 2015), a Índia (Duhan e Pandey, 2013), a China (Shao et al., 2015), a Sérvia (Gocic e Trajkovic, 2013), as Filipinas (Villafuerte II et al., 2014), o Brasil (Silva et al., 2016), entre outros.

A identificação de alterações nos registros meteorológicos é de grande importância para os estudos de engenharia que utilizam as séries históricas, pois tanto as simulações como as aplicações de teorias de probabilidade são realizadas com a hipótese de que as séries históricas são homogêneas, isto é, que não apresentam tendências. Em muitos casos são usados modelos estocásticos para realizar simulações hidrológicas, focados na predição e preparação da sociedade para os impactos provocados por possíveis eventos extremos ou raros (Moreira e Naghettini, 2016; Ishak et al., 2013).

A análise de tendências através de testes estatísticos não paramétricos vem sendo realizada por diversos pesquisadores visando identificar eventuais tendências monotônicas em séries hidrometeorológicas. Nestes estudos, os testes estatísticos não paramétricos de Mann-Kendall (MK) e Spearman's rho (SR) são amplamente utilizados para verificação da existência ou não de mudanças nas séries históricas.

Damé et al. (2013) analisaram 14 estações climatológicas no sul do estado do Rio Grande do Sul visando identificar a presença ou ausência de tendências nas séries históricas de precipitação total anual na bacia hidrográfica da Lagoa Mirim (lado brasileiro). Neste estudo foi utilizado o teste não paramétrico MK. Os autores observaram alterações em uma das estações e associaram o ocorrido com possível alterações devido às mudanças climáticas na região. Ávila et al. (2014) também utilizaram o teste MK para avaliar tendências das temperaturas mínimas e máximas em 43 localidades do estado de Minas Gerais. No Paquistão, Ahmad et al. (2015) analisaram possíveis alterações de tendências de precipitação em 15 estações utilizando os testes não paramétricos MK e SR. Os autores encontraram resultados semelhantes quanto aos testes não paramétricos.

De acordo com Moreira e Naghettini (2016), atenção especial deve ser dada na interpretação dos resultados de aumento ou decréscimo de tendências em variáveis hidrológicas. Muitos resultados encontrados na literatura justificam a variabilidade encontrada devido a fatores antrópicos. Entretanto, ainda segundo os autores, quando uma tendência realmente está presente em uma dessas variáveis, ela pode ter sido causada, dentre outros fatores, por fenômenos meteorológicos específicos como El Niño - Oscilação Sul (ENOS) (James, 1994), o qual é acoplamento dos fenômenos El Niño e La Niña que são caracterizados, respectivamente, pelo aquecimento e resfriamento anômalo das águas do Oceano Pacífico Equatorial central e leste.

O Brasil apresenta elevada vulnerabilidade aos possíveis efeitos das alterações climáticas (Silva et al., 2016). Para Assis et al. (2012), o cenário climático brasileiro acompanha a mesma tendência de aquecimento global, em que as mudanças mais significativas estão associadas às modificações nos padrões de chuva e alterações na distribuição de eventos climáticos extremos, como secas e inundações.

$\mathrm{O}$ leste da América do Sul, o qual se enquadra o estado do Rio Grande do Sul, está sob a influência do Sistema de Monções da América do Sul, sendo responsável pela formação de chuvas na região. Ainda segundo os autores, a região também é influenciada pela Zona de Convergência do Atlântico Sul, sendo caracterizada por uma zona baroclínica quase estacionária orientada na direção noroeste-sudeste, a qual se estende da região amazônica para o sul do Oceano Atlântico. Esta zona, quando ativa, está associada a nebulosidade e precipitações intensas (Bombardi et al., 2014).

$\mathrm{O}$ conhecimento da tendência da precipitação anual na metade norte do estado do Rio Grande do Sul tem se mostrado cada vez mais importante, principalmente devido à relação entre o aumento da precipitação e a produção de culturas a qual, segundo Berlato et al. (2005), pode estar associada ao fenômeno El Niño Oscilação Sul (ENOS). De acordo com Cera e Ferraz (2015), o Rio Grande do Sul situa-se em uma região latitudinal favorável à atuação de diversos fenômenos meteorológicos que afetam o tempo e o clima do Estado, podendo-se citar fenômenos de escala sinótica, como as frentes frias, as quais passam regularmente pelo Estado favorecendo a boa distribuição espacial e temporal das chuvas; fenômenos de escala intrassazonal, como a Oscilação de Madden e Julian (Ferraz, 2004); e fenômenos de escala global, como o El Niño e a La Niña. Grimm (2003) verificou a influência do El Niño na circulação monçônica de verão, concluindo que as anomalias de precipitação são favorecidas pela perturbação na circulação das Células de Walker e Hadley sobre o Oceano Pacífico Leste e América do Sul, e pelos trens de ondas de Rossby sobre o sul da América do Sul.

Sendo assim, o objetivo principal deste estudo foi relacionar as tendências de aumento e/ou decréscimo em séries temporais de precipitação na metade norte do estado do Rio Grande do Sul com as fases do ENOS. Os objetivos específicos do estudo foram: a) analisar a variabilidade média anual em séries temporais de precipitação 
através da regressão linear e dos testes de Mann-Kandall e Spearman's rho; b) selecionar eventos de El Niño e La Niña para relacionar com os períodos em foram detectadas tendências nas séries temporais de precipitação; e c) investigar quais as possíveis causas para as tendências encontradas nas séries temporais de precipitação.

\section{Material e Métodos}

Neste trabalho foram utilizados dados pluviométricos de oito estações meteorológicas da região norte do estado do Rio Grande do Sul (Fig. 1), obtidas na plataforma Hidroweb, da base de dados da Agência Nacional de Águas - ANA.

Os dados de precipitação foram analisados por meio do software SYHDA (System of Hydrological Data Acquisition and Analysis) (Vargas et al., 2019), no qual foi possível obter os totais anuais de precipitações e, em seguida, foram selecionadas as estações com menos de 15 dias de falhas (Tabela 1). Nos anos que foram detectadas falhas realizou-se o preenchimento através do método do vetor de ponderação regional (Tucci, 2004).

Na sequência, por meio do software SigmaPlot v.10.0, a distribuição temporal da precipitação total anual de cada uma das estações foi plotada em forma de gráfico, juntamente com a média e a regressão linear, com intuito de realizar uma análise qualitativa dos dados de preci-
Tabela 1 - Dados das estações pluviométricas utilizadas no estudo.

\begin{tabular}{lccccc}
\hline Código & Nome & $\begin{array}{c}\text { Latitude } \\
(\mathrm{S})\end{array}$ & $\begin{array}{c}\text { Longitude } \\
(\mathrm{W})\end{array}$ & $\begin{array}{c}\text { Altitude } \\
(\mathrm{m})\end{array}$ & $\begin{array}{c}\mathrm{N} \\
(\mathrm{anos})\end{array}$ \\
\hline 02850009 & $\begin{array}{c}\text { Passo das } \\
\text { Tainhas }\end{array}$ & $\begin{array}{c}28^{\circ} \\
52^{\prime} 03^{\prime \prime}\end{array}$ & $50^{\circ} 27^{\prime} 22^{\prime \prime}$ & 640 & 46 \\
02851003 & Antônio Prado & $28^{\circ}$ & $51^{\circ} 17^{\prime} 04^{\prime \prime}$ & 630 & 46 \\
& $51^{\prime} 12^{\prime \prime}$ & & & \\
02851022 & $\begin{array}{c}\text { Passo } \\
\text { Migliavaca }\end{array}$ & $\begin{array}{c}28^{\circ} \\
37^{\prime} 10^{\prime \prime}\end{array}$ & $51^{\circ} 52^{\prime} 00^{\prime \prime}$ & 380 & 46 \\
02851024 & Prata & $28^{\circ}$ & $51^{\circ} 37^{\prime} 42^{\prime \prime}$ & 680 & 46 \\
02854005 & $\begin{array}{c}\text { Passo Major } \\
\text { Zeferino }\end{array}$ & $\begin{array}{c}28^{\circ} \\
44^{\prime} 01^{\prime \prime}\end{array}$ & $54^{\circ} 38^{\prime} 52^{\prime \prime}$ & 160 & 32 \\
02854011 & São Luiz & $28^{\circ}$ & $54^{\circ} 58^{\prime} 00^{\prime \prime}$ & 245 & 41 \\
& Gonzaga & $24^{\prime} 00^{\prime \prime}$ & & & \\
02951010 & Encantado & $29^{\circ}$ & $51^{\circ} 51^{\prime} 34^{\prime \prime}$ & 60 & 60 \\
& & $14^{\prime} 16^{\prime \prime}$ & & & \\
02951022 & Nova Palmira & $29^{\circ}$ & $51^{\circ} 11^{\prime} 25^{\prime \prime}$ & 80 & 60 \\
& & $20^{\prime} 06^{\prime \prime}$ & & & \\
\hline
\end{tabular}

pitação e observar possíveis tendências mais evidentes nos dados.

A variabilidade pluviométrica foi comparada com os períodos de ocorrência de El Niño e La Niña, descritos por Berlato et al. (2005). De acordo com os autores, os dados são referentes ao período de julho do ano de início de fenômeno a junho do ano seguinte (El Niño: 1925-1926,1929-1930, 1930-1931, 1940-1941,

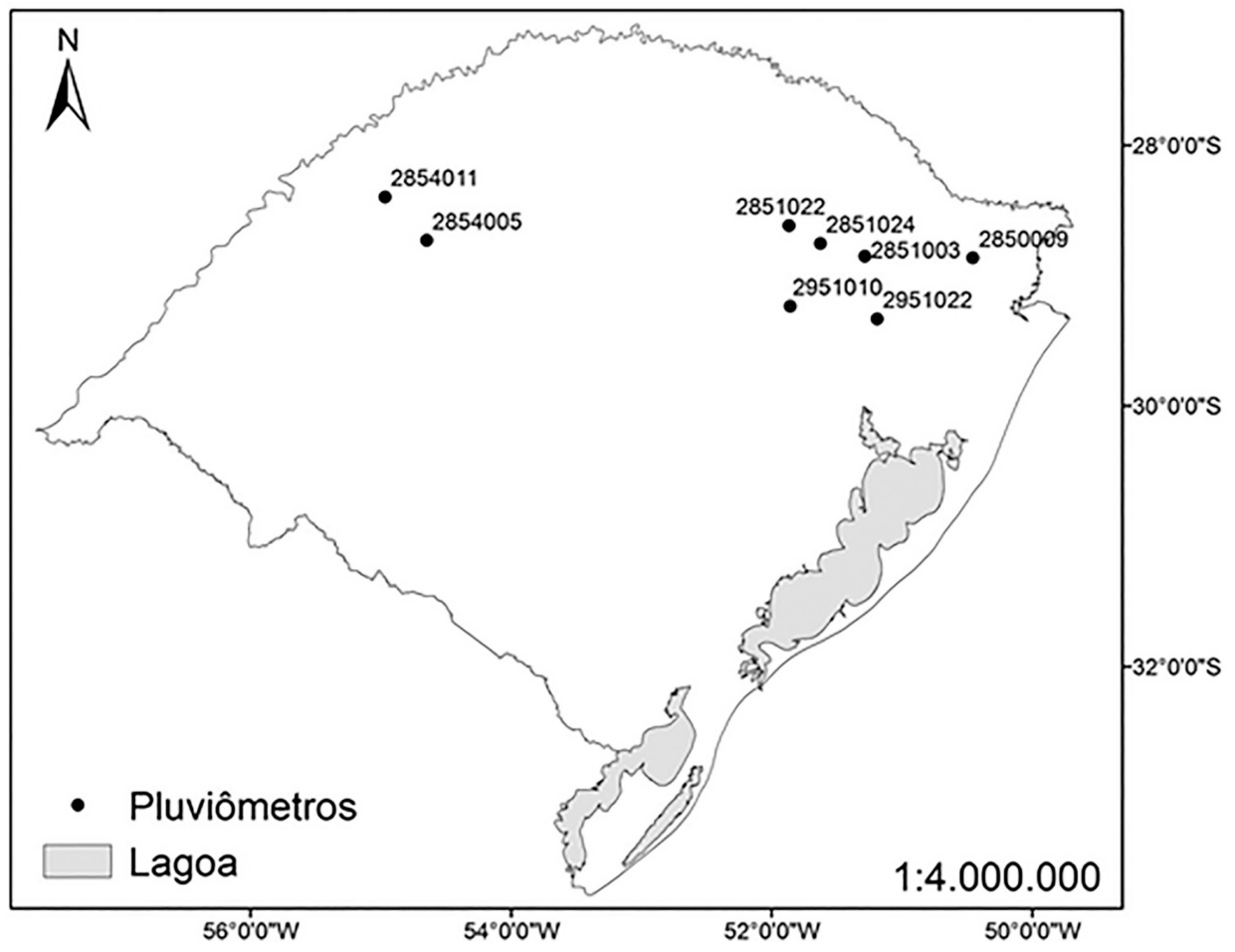

Figura 1 - Mapa do estado do Rio Grande do Sul com a localização das estações pluviométricas. 
1951-1952, 1957-1958, 1963-1964, 1965-1966, 1969-1970, 1972-1973,1976-1977, 1982-1983, 1986-1987, 1987-1988, 1991-1992, 1992-1993, 1993-1994, 1994-1995, 1997-1998,2002-2003; La Niña: 1922-1923, 1924-1925, 1938-1939, 1942-1943, 1944-1945, 1949-1950, 1954-1955,1955-1956, 1956-1957, 1964-1965, 1967-1968, 1970-1971, 1971-1972, 1973-1974, 1974-1975, 1975-1976, 1988-1989, 1995-1996, 1998-1999, 1999-2000).

Após identificação visual aplicou-se os testes estatísticos não paramétricos Mann-Kendall e Spearman's rho, por meio do software TREND v. 1.0.2 (Chiew e Siriwardena, 2005).

O teste estatístico não paramétrico de Mann-Kendall (Mann, 1945; Kendall, 1975) verifica se há uma tendência nos dados de séries temporais. Consiste em comparar cada valor da série temporal com os valores restantes, sempre em ordem sequencial, sendo contado o número de vezes em que os termos restantes são maiores que o valor analisado. A estatística $\mathrm{S}$ é obtida pela soma de todas as contagens, onde o sinal $\left(\mathrm{x}_{\mathrm{i}}-\mathrm{x}_{\mathrm{j}}\right)$ é determinado por:

$$
S=\sum_{i=2}^{n} \sum_{j=1}^{i-1} \operatorname{sgn}\left(x_{i}-x_{j}\right)
$$

A estatística $\mathrm{S}$ tende para a normalidade quando $\mathrm{n}$ é grande,

$$
\operatorname{sgn}=\left\{\begin{array}{l}
1 \text { se }\left(\mathrm{x}_{\mathrm{i}}-\mathrm{x}_{\mathrm{j}}\right)>0 \\
0 \text { se }\left(\mathrm{x}_{\mathrm{i}}-\mathrm{x}_{\mathrm{j}}\right)=0 \\
-1 \text { se }\left(\mathrm{x}_{\mathrm{i}}-\mathrm{x}_{\mathrm{j}}\right)<0
\end{array}\right.
$$

com média $\mathrm{E}(\mathrm{S})$ e variância $\operatorname{Var}(\mathrm{S})$ definidas como seguem:

$$
\begin{aligned}
& \mathrm{E}(\mathrm{S})=0, \\
& \operatorname{Var}(\mathrm{S})=\frac{\mathrm{n}(\mathrm{n}-1)(2 \mathrm{n}+5)-\sum_{\mathrm{p}=1}^{\mathrm{q}} \mathrm{t}_{\mathrm{p}}\left(\mathrm{t}_{\mathrm{p}}-1\right)\left(2 \mathrm{t}_{\mathrm{p}}+5\right)}{18}
\end{aligned}
$$

em que, $\left(\mathrm{t}_{\mathrm{p}}\right)$ é o número de dados com valores iguais em certo grupo (pth) e q é o número de grupos contendo valores iguais na série de dados em um grupo p. O segundo termo da Eq. 3 representa um ajuste para a variância dos dados.

Conforme Ahmad et al. (2015), um valor positivo de $\mathrm{S}$ na Eq. 2 indica tendência positiva na qual os dados crescem com o tempo; um valor negativo de $\mathrm{S}$ indica uma tendência negativa. Sabendo-se que $\mathrm{S}$ normalmente distribuído tem média zero e variância dada pela Eq. 3, pode-se verificar se a tendência positiva ou negativa é significantemente diferente de zero. Se $\mathrm{S}$ é significantemente diferente de zero, a hipótese nula $\left(\mathrm{H}_{\mathrm{o}}\right)$ pode ser rejeitada para certo nível de significância, apontando para a existência de tendência (hipótese alternativa $\mathrm{H}_{1}$ é aceita).

Mann (1945) e Kendall (1975) mostraram que mesmo para valores pequenos de $\mathrm{n}$, pode-se assumir uma distribuição normal desde que o teste estatístico parametrizado $Z_{\text {calc }}$ seja dado por:

$$
\mathrm{Z}_{\text {calc }}\left\{\begin{array}{lc}
\frac{\mathrm{S}-1}{\sqrt{\operatorname{Var}(\mathrm{S})}} & \text { se } \mathrm{S}>0 \\
0 & \text { se } \mathrm{S}=0 \\
\frac{\mathrm{S}+1}{\sqrt{\operatorname{Var}(\mathrm{S})}} & \text { se } \mathrm{S}<0
\end{array}\right.
$$

A presença de tendência estatisticamente significativa na série temporal é avaliada através do valor de $Z_{\text {calc }}$; esta estatística é usada para testar a hipótese nula de que nenhuma tendência existe. $O$ valor positivo de $Z_{\text {calc }}$ indica tendência crescente. Para testar qualquer tendência constante, crescente ou decrescente para um determinado nível de significância $p$, a hipótese nula é rejeitada se o valor absoluto de $Z_{\text {calc }}$ é maior que $Z_{\text {tab }}$, obtido na tabela de distribuição normal (Santos et al., 2010).

O Spearman's rho (Lehmann, 1975) é um teste estatístico não paramétrico baseado em classificação, usado para análise de tendência. Neste teste, assume-se que os dados da série temporal são independentes e igualmente distribuídos. A hipótese nula $\left(\mathrm{H}_{0}\right)$ indica ausência de tendência ao longo do tempo e a hipótese alternativa $\left(\mathrm{H}_{1}\right)$ indica que há tendência monotônica nos dados, podendo apresentar aumento ou decréscimo nos dados da série temporal (Yue et al., 2002). O teste estatístico $\mathrm{R}_{\mathrm{sp}}$ e a estatística normalizada $\mathrm{Rho}_{\text {calc }}$ são definidos como (Ahmad et al., 2015):

$$
\begin{gathered}
\mathrm{R}_{\mathrm{sp}}=1-\frac{6 \sum_{\mathrm{i}=1}^{\mathrm{n}}\left(\mathrm{D}_{\mathrm{i}}-\mathrm{i}\right)^{2}}{\mathrm{n}\left(\mathrm{n}^{2}-1\right)} \\
\mathrm{Rho}_{\text {calc }}=\mathrm{R}_{\mathrm{sp}} \sqrt{\frac{\mathrm{n}-2}{1-\mathrm{R}_{\mathrm{sp}}^{2}}}
\end{gathered}
$$

em que, $D_{\mathrm{i}}$ é o posicionamento da informação na série temporal para i observações, i é o número da ordem cronológica, $\mathrm{n}$ é o número total de dados na série, e $\mathrm{Rho}_{\text {calc }}$ é a distribuição t de Student com $(n-2)$ graus de liberdade.

Os valores positivos de $\mathrm{Rho}_{\text {calc }}$ representam um aumento de tendência na série histórica dos dados hidrometeorológicos; valores negativos representam tendências decrescentes. Esses valores são comparados com os valores de $\mathrm{Rho}_{\text {tab }}$, os quais são definidos na tabela de distribuição $\mathrm{t}$ de Student para um determinado nível de significância $\mathrm{p}$. Se o valor absoluto de $\mathrm{Rho}_{\text {calc }}$ for maior que $\mathrm{Rho}_{\text {tab }}, \mathrm{H}_{0}$ é rejeitada e existe uma tendência significativa na série temporal (Ahmad et al., 2015). 


\section{Resultados e Discussões}

Na Fig. 2 estão apresentadas as séries temporais dos dados de precipitação total anual, a média e a regressão linear das oito estações pluviométricas utilizadas no estudo.
Observa-se graficamente, por meio da curva de regressão linear, que as estações 02850009, 02851003, 02851022, 02851024, 02951010 e 02951022 apresentaram tendência de aumento nos dados de chuva com o passar do tempo. Este resultado pode ser explicado em função de eventos meteorológicos comumente presentes

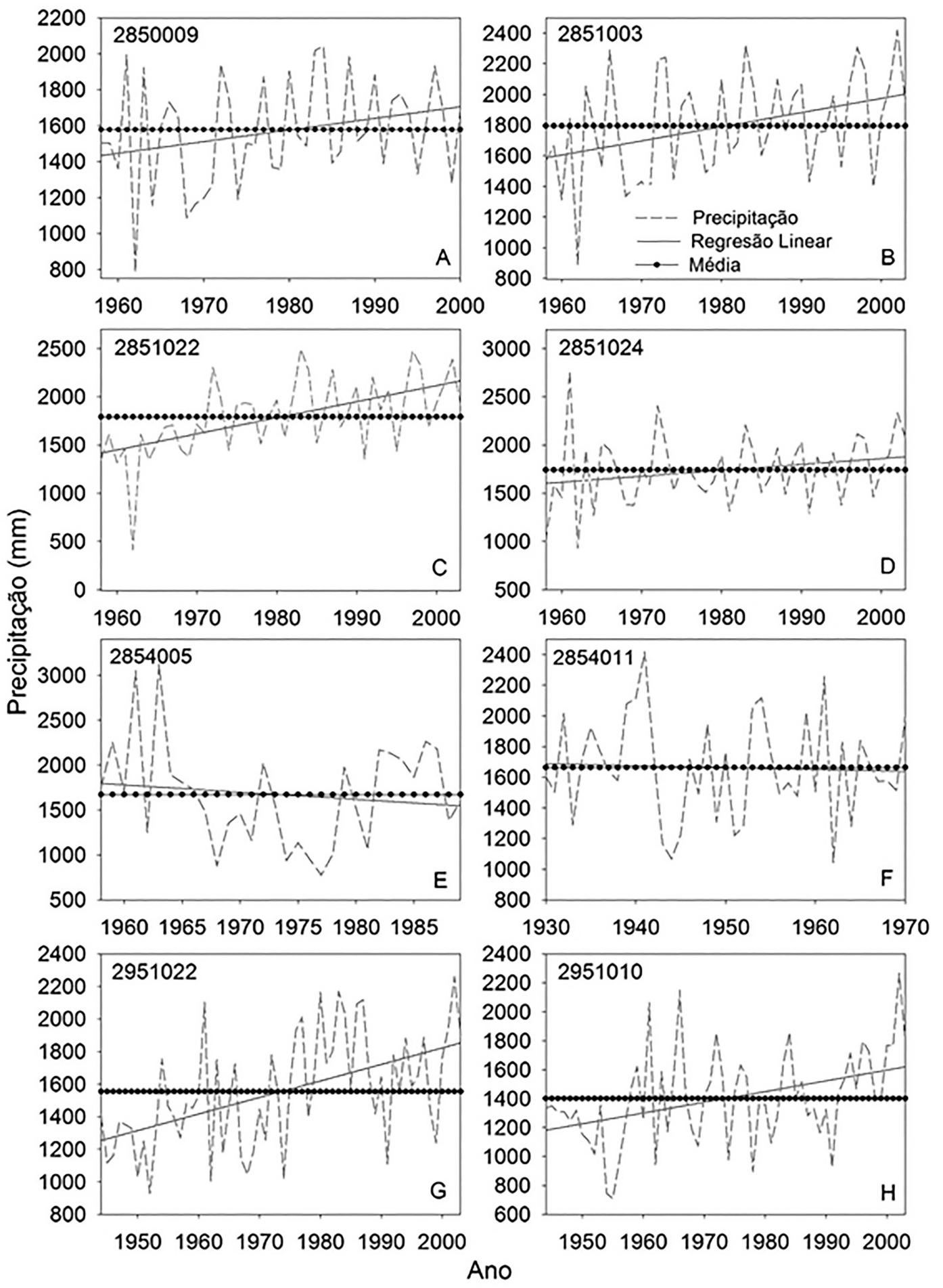

Figura 2 - Precipitação total anual, média e regressão linear de oito estações meteorológicas do norte do estado do Rio Grande do Sul. 
no estado do Rio Grande do Sul. De acordo com Campos e Eichholz (2011), o Estado sofre a influência da passagem dos Sistemas Frontais (SF) e dos Jatos de Baixos Níveis (JBN), que transportam calor e umidade da Amazônia para o sul do Brasil. Além do mais, ainda segundo Campos e Eichholz (2011), a região onde o Rio Grande do Sul se localiza possui características ideais para o desenvolvimento de atividade convectiva de precipitação e formação e manutenção de Sistemas Convectivos de Mesoescala (SCM), uma vez que existe uma possível correlação entre a ocorrência de nascimentos de SCM e a topografia, que pode estar relacionada com efeitos térmicos e orográficos que geram esse tipo de sistemas. Para Sakamoto (2009), a formação dos SCM no norte do Rio Grande do Sul pode ter como possíveis causadores a convergência noturna no vale do rio Paraná, o fluxo de umidade oceânica associado à circulação da Alta do Atlântico Sul (associada à circulação média meridional da atmosfera, que surge devido à célula de Hadley), as frentes frias e a Zona de Convergência do Atlântico Sul.

A estação 02854005 apresentou uma leve tendência de diminuição da precipitação total com o passar dos anos e a única estação que se mostrou indiferente a tendências nos dados foi à estação 02854011. Oliveira e Silva (2016), analisando a variabilidade climática do estado do Rio Grande Sul, identificou quatro regiões homogêneas quanto às anomalias anuais de precipitação. Em seus resultados observaram que as estações pluviométricas localizadas na porção noroeste do Estado (02854005 e 02854011) foi a região em que foram observadas as maiores diferenças de precipitação de um ano para outro, no período compreendido entre 1981 a 2006, indicando que essa região do Estado é uma das que mais estão sob a influência das forçantes climáticas que atuam na escala interanual, dentre eles a formação de SCM.

No ano de 2002, nas estações pluviométricas de código 02851003, 02951022 e 02951010 , verificou-se um pico de precipitação total anual, possivelmente associado à ocorrência do fenômeno El Niño, conforme descrito em Berlato et al. (2005). Na estação 02854005 , no ano de 1963, também ocorreu um pico de precipitação, provavelmente devido ao mesmo fenômeno. O mesmo ocorreu nas estações 02854011,0281024 e 02850009 , nas quais o aumento brusco da precipitação total aconteceu nos anos 1941, 1972 e 1983/1984, respectivamente.

Este trabalho confirmou alguns períodos em que ocorreram anomalias climatológicas apresentado por Berlato et al. (2005). A estação 02854005 apresentou período significativo de escassez hídrica entre os anos de 1967 a 1978. Igualmente aconteceu com as estações $02850009 \mathrm{e}$ 02851003, uma vez que a precipitação total foi abaixo da média neste período, a qual, segundo Nimer (1979), seria igual a $1500 \mathrm{~mm}$ para a metade norte do estado do Rio Grande do Sul. Uma das justificativas é a ocorrência do efeito La Niña nesses intervalos de anos, que pode ter afetado o regime de chuvas nessas estações. A La Niña também pode ter causado a redução na precipitação total nos anos de 1944 (02854011), 1954 (02951010) e 1999 (2851003).

Estes resultados vêm confirmar os resultados encontrados por Berlato et al. (2005), o qual analisou a associação entre fenômenos de alteração global com a produtividade de milho no estado do Rio Grande do Sul. Nos últimos anos, é crescente os trabalhos que relacionam a precipitação com anomalias de Temperatura da Superfície do Mar (TSM) no Atlântico Sul, inclusive no sul do Brasil (Bombardi et al., 2014). Entretanto, é importante ressaltar que existem fatores geomorfológicos, assim como fatores antrópicos, que também podem tornar a precipitação irregular.

Segundo Robaina et al. (2016), o norte do estado do Rio Grande do Sul é caracterizado por encostas com bases amplas, como topos estreitos e alongados que se associam a ressaltos e cristas laterais. Esta topografia montanhosa favorece as chuvas orográficas, onde uma massa de ar úmida é impedida por uma forma íngreme do relevo, como uma montanha, serra ou escarpa. Neste encontro ocorrem fortes chuvas, que retiram toda a umidade do ar (Tucci, 2004).

A produção de milho no norte do estado do Rio Grande do Sul assume uma grande importância na agricultura local, sendo, de acordo com Ribeiro (2003), como a segunda maior cultura agrícola do Estado, em termos de área cultivada, e a terceira em produção de grãos. Este fato aumenta a importância de se analisar a influência das anomalias climáticas no regime de chuvas na região, e, ao mesmo tempo, é mais um fator que pode aumentar naturalmente o regime de chuvas, em função do aumento da evapotranspiração potencial (Tucci, 2004). Ou seja, a tendência de aumento de precipitação em uma determinada série meteorológica não pode ser diretamente associada ao fenômeno El Niño; é preciso analisar outros fatores, como variáveis hidrológicas, uso e ocupação do solo, fatores antrópicos, conforme indicado por Uliana et al. (2015).

Os resultados qualitativos apresentados nos gráficos da Fig. 2 foram comparados com a análise estatística não paramétrica. Na Tabela 2 estão apresentados os resultados das análises de tendências feitos pelos testes Mann-Kendall (MK) e Spearman's rho (SR).

Observa-se na Tabela 2 que ambos os resultados dos testes estatísticos foram idênticos, não indicando divergências nas análises. Resultados similares foram obtidos por Yue et al. (2002), os quais avaliando a tendência de séries hidrológicas no Canadá também encontraram resultados idênticos para os dois testes. Além disso, Ahmad et al. (2015) analisando a tendência das precipitações em uma bacia hidrográfica no Paquistão verificaram concordância entre os resultados dos testes MK e SR para dados mensais, sazonais e anuais de precipitação. Entretanto, Cera e Ferraz (2015) encontraram divergências de 
Tabela 2 - Resultados do teste de Mann-Kendall e Spearman's rho para as séries de precipitação total anual.

\begin{tabular}{|c|c|c|c|c|c|c|c|c|}
\hline \multirow[t]{2}{*}{ Estação } & \multicolumn{4}{|c|}{ Mann-Kendall } & \multicolumn{4}{|c|}{ Spearman's rho } \\
\hline & $Z_{\text {calc }}$ & $\mathrm{Z}_{\mathrm{tab}}$ & $\mathrm{H}_{0}(\mathrm{Z})$ & Tendência & $\mathrm{Rho}_{\text {calc }}$ & $\mathrm{Rho}_{\text {tab }}$ & $\mathrm{H}_{0}(\mathrm{w})$ & Tendência \\
\hline 02850009 & 1,922 & 1,96 & A & - & 1,886 & 1,96 & A & - \\
\hline 02851003 & 2,197 & 1,96 & $\mathrm{R}$ & $\uparrow$ & 2,106 & 1,96 & $\mathrm{R}$ & $\uparrow$ \\
\hline 02851022 & 4,128 & 1,96 & $\mathrm{R}$ & $\uparrow$ & 3,917 & 1,96 & $\mathrm{R}$ & $\uparrow$ \\
\hline 02851024 & 1,856 & 1,96 & A & - & 1,818 & 1,96 & A & - \\
\hline 02854005 & $-0,243$ & 1,96 & A & - & $-0,163$ & 1,96 & A & - \\
\hline 02854011 & $-0,168$ & 1,96 & A & - & $-0,371$ & 1,96 & A & - \\
\hline 02951010 & 3,246 & 1,96 & $\mathrm{R}$ & $\uparrow$ & 3,396 & 1,96 & $\mathrm{R}$ & $\uparrow$ \\
\hline 02951022 & 4.165 & 1,96 & $\mathrm{R}$ & $\uparrow$ & 4,111 & 1,96 & $\mathrm{R}$ & $\uparrow$ \\
\hline
\end{tabular}

A: aceita a hipótese nula $\left(\mathrm{H}_{0}\right)$ a $\mathrm{p}<0,05$; $\mathrm{R}$ : rejeita a hipótese nula $\left(\mathrm{H}_{0}\right)$ a $\mathrm{p}<0,05$.

resultados ao aplicar os testes não paramétricos de Run, o qual não apresentou tendência, e Pettit, o qual apresentou tendência, em séries de dados pluviométricos para o estado do Rio Grande do Sul. Para confirmar qual hipótese estava correta, as autoras aplicaram o teste de MK, o qual indicou que realmente as séries não eram homogêneas. Concluíram alertando da importância em se aplicar mais de um teste de tendência para não correr o risco de realizar uma análise incorreta.

De acordo com os resultados dos testes estatísticos não paramétricos $\mathrm{MK}$ e SR as estações 02850009, 02851024,02854005 e 02854011 não apresentaram tendência significativa a $p<0,05$. Este resultado contrasta, em parte, com os resultados encontrados na análise qualitativa da variabilidade temporal dos dados pluviométricos apresentados na Fig. 2. Nos gráficos, a estação 02854011 (Fig. 2-F) foi a que apresentou menor variabilidade temporal, indicando inicialmente que essa variabilidade poderia ser um reflexo das alterações do ciclo hidrológico ao longo do tempo. Nos mesmos gráficos, a tendência decrescente apresentada pela Fig. 2-E não foi confirmada pelas análises estatísticas, o que indica também uma variabilidade natural dos dados pluviométricos ao longo do tempo.

De acordo com Damé et al. (2013) fatores antrópicos, como o desmatamento e o uso indevido dos ecossistemas, podem modificar o clima de uma região contribuindo para as mudanças no sistema global. Berlato et al. (2005), estudando dados de precipitação de 17 locais do Rio Grande do Sul, não identificaram tendências de longo prazo na precipitação pluvial anual, ressaltando que as reduções e aumentos que se observaram em curto período se referem, possivelmente, à flutuação natural da precipitação pluvial. Fontana e Berlato (1997) mostraram que nos anos de ocorrência de El Niño são registrados no Rio Grande do Sul valores de precipitação pluvial acima da média no período de outubro a dezembro. Esta hipótese reforça a ideia de que a mudança na precipitação seja apenas uma oscilação de causa natural, não necessariamente associada a uma mudança definitiva nos padrões pluviométricos.
Para as estações 02851003, 02851022, 02951010 e 02951022, os resultados das análises estatísticas corroboram os resultados encontrados pelos gráficos, indicando uma tendência significativa de aumentos de chuvas ao longo dos anos. Isto demonstra que $50 \%$ das estações pluviométricas analisadas no norte do estado do Rio Grande do Sul apresentaram aumento dos índices pluviométricos ao longo dos anos. Resultados similares foram encontrados por Haylock et al. (2006), Sansigolo e Kayano (2010) e Cera e Ferraz (2015), estudos esses realizados no estado do Rio Grande do Sul.

De acordo com Berlato et al. (2005), o excesso ou a ausências de chuvas é um fator determinante na produtividade e no manejo de diversas culturas, sendo a cultura do milho, objeto de estudos dos autores, influenciado positivamente (aumento de produtividade) pelo fenômeno El Niño, onde acontece um aumento de chuvas na região, e negativamente pela La Niña, onde há um decréscimo dos índices pluviométricos.

\section{Conclusões}

De acordo com os resultados obtidos pode-se concluir que:

1. O aumento da precipitação total anual nas séries temporais pode estar relacionado com os períodos de El Niño e La Niña. Entretanto, estudos mais específicos devem ser realizados para confirmar essa hipótese, uma vez que a variabilidade de precipitação também pode estar relacionada com alterações hidrológicas, em função do uso e ocupação do solo, e com atividades antrópicas na região norte do Estado.

2. Os testes não paramétricos Mann-Kendall e Spearman's rho apresentaram concordância nas análises de tendência da precipitação total anual no norte do estado do Rio Grande do Sul.

3. Os testes Mann-Kendall e Spearman's rho identificaram mudanças na tendência de $50 \%$ das estações pluviométricas, indicando um aumento na precipitação 
total anual na região norte do estado do Rio Grande do Sul.

\section{Referências}

AHMAD, I.; TANG, D.; WANG, T. F.; WANG, M.; WANG, B. Precipitation trends over time using Mann-Kendall and Spearman's rho tests in Swat river basin, Pakistan. Advances in Meteorology, v. 2016, 431860, 2015.

ASSIS, J.M.O.; LACERDA, F.F.; SOBRAL, M.C.M. Análise de detecção de tendências no padrão pluviométrico na bacia hidrográfica do Rio Capibaribe. Revista Brasileira de Geografia Física, v. 2, p. 320-331, 2012.

ÁVILA, L.F.; MELLO, C.R.; YANAGI, S.N.M.; SACRAMENTO NETO, O.B. Tendências de temperaturas mínimas e máximas do ar no estado de Minas Gerais. Pesquisa Agropecuária Brasileira, v. 49, n. 4, p. 247-256, 2014.

BERLATO, M.A.; FARENZENA, H.; FONTANA, D.C. Associação entre El Niño Oscilação Sul e a produtividade do milho no estado do Rio Grande do Sul. Pesquisa Agropecuária Brasileira, v. 40, n. 5, p. 423-432, 2005.

BOMBARDI, R.J.; CARVALHO, L.M.V.; JONES, C.; REBOITA, M.S. Precipitation over eastern South America and the South Atlantic Sea surface temperature during neutral ENSO periods. Climate Dynamics, v. 42, p. 1553-1568, 2014.

CAMPOS, C.R.J.; EICHHOLZ, C.W. Características físicas dos Sistemas Convectivos de Mesoescala que afetaram o Rio Grande do Sul no período de 2004 a 2008. Revista Brasileira de Geofísica, v. 29, n. 2, p. 331-345, 2011.

CERA, J.C.; FERRAZ, S.E.T. Variações climáticas na precipitação no sul do Brasil no clima presente e futuro. Revista Brasileira de Meteorologia, v. 30, n. 1, p. 81-88, 2015.

CHIEW, F.; SIRIWARDENA, L. Cooperative Research Centre for Catchment Hydrology Trend User Guide. Camberra: E-water., 2005. 29 p. Disponível em: http://www.toolkit. net.au/Tools/TREND/documentation. Acesso em: jun. 2019.

DAMÉ, R.C.F.; TEIXEIRA, C.F.A.; BACELAR, L.C.S.; WINKLER, A.S.; SANTOS, J.P. Montonic trend and change points in southern Brazil precipitation. Engenharia Agrícola, v. 33, n. 2, p.258-268, 2013.

DUHAN, D.; PANDEY, A. Statistical analysis of long term spatial and temporal trends of precipitation during 1901-2002 at Madhya Pradesh, India. Atmospheric Research, v. 122, p. 136-149, 2013.

FERRAZ, S.E.T. Variabilidade intrasazonal no Brasil e sul da América do Sul. São Paulo, 2004. Tese (Doutorado em Meteorologia) - Universidade de São Paulo, São Paulo, $145 \mathrm{p}$.

FONTANA, D.C.; BERLATO, M.A. Influência do El Niño: oscilação sul sobre a precipitação pluvial do estado do Rio Grande do Sul. Revista Brasileira de Agrometeorologia, v. 5, n. 1, p. 127-132, 1997.

GOCIC, M.; TRAJKOVIC, S. Analysis of precipitation and drought data in Serbia over the period 1980-2010. Journal of Hydrology, v. 494, p. 32-42, 2013.
GRIMM, A.M. The El Niño impact on the summer monsoon in Brazil: regional processes versus remote influences. Journal of Climate, v. 16, n. 2, p. 263-280, 2003.

HAYLOCK, M.R.; PETERSON, T.C.; ALVES, L.M.; AMBRIZZI, T.; ANUNCIAÇÃO, Y.M.T. et al. Trends in Total and Extreme South American Rainfall in 1960-2000 and Links with Sea Surface Temperature. Journal of Climate, v. 19, p. 1490-1512, 2006.

ISHAK, E.H.; RAHMAN, A.; WESTRA, S.; SHARMA, A.; KUCZERA, G. Evaluating the non-stationarity of Australian annual maximum flood. Journal of Hydrology, v. 494, p. 134-145, 2013.

JAMES, I.N. Introduction to Circulating Atmospheres. Cambridge Atmospheric and Space Science, 1994, 422p.

KENDALL, M.G. Rank Correlation Methods. Griffin, London, 1975.

LEHMANN, E.L. Nonparametrics, Statistical Methods Based on Ranks. Holden-Day: San Francisco, 1975.

MANN, H.B. Nonparametric tests against trend. Econometrica, v.13, n. 3, p.245-259, 1945.

MOREIRA, J.G.V.; NAGHETTINI, M. Detecção de tendências monotônicas temporais e relação com erros dos tipos I e II: estudo de caso em séries de precipitações diárias máximas anuais do estado do Acre. Revista Brasileira de Meteorologia, v. 31, n. 4, p. 394-402, 2016.

NIMER, E. Climatologia do Brasil. Rio de Janeiro: IBGE. 422p. 1979.

OLIVEIRA, K.S.S.; SILVA, M.G.A.J. Onda circumpolar antártica: Influência na variabilidade climática do estado do Rio Grande do Sul. Revista Brasileira de Meteorologia, v. 31, n. 4, p. 403-414, 2016.

POLZIN, D.; HASTENRATH, S. Climate of Brazil's nordeste and tropical atlantic sector: preferred time scales of variability. Revista Brasileira de Meteorologia, v. 29, n. 2, p. 153-160, 2014.

RIBEIRO, R.E.P.; ÁVILA, P.L.R.; BRITO, J.I.B.; SANTOS, E. G.; SOUSA, L.F. Análise da tendência climática nas séries temporais de temperatura e precipitação de Tucuruí-Pará. Revista Brasileira de Geografia Física, v. 7, n. 5, p. 798807, 2014.

RIBEIRO, A.S. Fisionomia agropecuária: culturas. Porto Alegre: Emater, 2003. 43p. (Caderno 2). Disponível em: http:// www.emater.tche.br. Acesso em: abr. 2018.

ROBAINA, L.E.S.; TRENTIN, R.; LAURENT, F. Compartimentação do estado do Rio Grande do Sul, Brasil, através do uso de Geomorphons obtidos em classificação topográfica automatizada. Revista Brasileira de Geomorfologia, v. 17, n. 2, p. 287-298, 2016.

SAKAMOTO, M.S. Sistemas convectivos de mesoescala observados na região subtropical da América do Sul durante o SALLJEX. São Paulo, 2009. Tese (Doutorado em Meteorologia) - Universidade de São Paulo, São Paulo, $243 p$.

SANSIGOLO, C.A.; KAYANO, M.T. Trends of seasonal maximum and minimum temperatures and precipitation in Southern Brazil for the 1913-2006 period. Theoretical and Applied Climatology, v. 101, n. 1-2, p. 209-216, 2010.

SANTOS, D.N.; SILVA, V.P.R.; SOUZA, F.A.S.; SILVA, R.A. Estudos de alguns cenários climáticos para o Nordeste do 
Brasil. Revista Brasileira de Engenharia Agrícola e Ambiental, v. 14, n. 5, p. 492-500, 2010.

SHAO, Y.; WU, J.; YE, J.; LIU, Y. Frequency analysis and its spatiotemporal characteristics of precipitation extreme events in China during 1951-2010. Theoretical and Applied Climatology, v. 121, p. 775-787, 2015.

SILVA, F.B.; SANTOS, J.R.N.; FEITOSA, F.E.C.S.; SILVA, I.D. C.; ARAÚJO, M.L.S. et al. Evidências de mudanças climáticas na região de transição Amazônica-Cerrado no estado do Maranhão. Revista Brasileira de Meteorologia, v. 31, n. 3, p. 330-336, 2016.

TUCCI, C.E.M. Hidrologia: Ciência e Aplicação, 3a ed. Porto Alegre: Editora Universidade/UFRGS: ABRH, 943 p., 2004.

ULIANA, E.M.; SILVA, D.D.; ULIANA, E.M.; RODRIGUES, B.S.; CORRÊDO, L.P. Análise de tendência em séries históricas de vazão e precipitação: uso de teste estatístico não paramétrico. Revista Ambiente \& Água, v. 10, n. 1, p. 8288, 2015.

VARGAS, M.M.; BESKOW, S.; CALDEIRA, T.L.; CORRÊA, L.L.; CUNHA, Z.A. SYHDA - System of Hydrological Data Acquisition and Analysis. Revista Brasileira de Recursos Hídricos, v. 24, e11, p. 1-10, 2019.

VILLAFUERTE II, M.Q.; MATSUMOTO, J.; AKASAKA, I.; TAKAHASHI, H.G.; KUBOTA, H. et al. Long-term trends and variability of rainfall extremes in the Philippines. Atmospheric Research, v. 137, p. 1-13, 2014.

YUE, S.; PILON, P.; CAVADIAS, G. Power of the Mann-Kendall and Spearman's rho tests for detecting monotonic trends in hydrological series. Journal of Hydrology, v. 264, p. 262-263, 2002.

This is an Open Access article distributed under the terms of the Creative Commons Attribution License, which permits unrestricted use, distribution, and reproduction in any medium, provided the original work is properly cited. 